\title{
INVARIANT MEASURES FOR TYPICAL CONTINUOUS MAPS ON MANIFOLDS
}

\author{
ELEONORA CATSIGERAS AND SERGE TROUBETZKOY
}

\begin{abstract}
We study the invariant measures of typical $C^{0}$ maps on compact connected manifolds with or without boundary, and also of typical homeomorphisms. We prove that the weak* closure of the set of ergodic measures coincides with the weak* closure of the set of measures supported on periodic orbits and also coincides with the set of pseudo-physical measures. Furthermore, we show that this set has empty interior in the set of invariant measures.
\end{abstract}

\section{INTRODUCTION}

In this article we study the structure of the invariant measures for typical continuous maps of a $C^{1}$ compact, connected manifold $M$ of finite dimension $m \geq 1$, with or without boundary. This generalizes the work in our previous article [CT] where we studied the case when $M$ is an interval. The study of the invariant measures for typical maps was initiated recently by Abdenur and Andersson $\mathrm{AA}$. Previously studies of the dynamics of typical maps have concentrated on the topological properties; see [AHK, $\mathrm{AP}, \mathrm{H1},[\mathrm{H} 2,[\mathrm{KMOP}, \mathrm{O}, \mathrm{OU}, \mathrm{PP}, \mathrm{Y}]$ and the references therein.

Let $\mathcal{E}_{f}$ denote the set of ergodic, $f$-invariant Borel probability measures, $\mathrm{Per}_{f}$ the set of invariant measures supported on a single periodic orbit, and $\mathcal{O}_{f}$ denote the set of pseudo-physical measures for $f$, (see subsection 2.1 for the definition). We always have $\operatorname{Per}_{f} \subset \mathcal{E}_{f}$. Let $\mathcal{C}(M)$ denote the set of continuous maps of $M$ to itself and $\mathcal{H}(M)$ denote the set of homeomorphisms of $M$ to itself. We endow these spaces with the $C^{0}$ topology and say that a family of maps in $\mathcal{C}(M)$ (resp. in $\mathcal{H}(M)$ ) is typical if it contains a countable intersection of open and dense family. Our main result is the following theorem:

We gratefully acknowledge support of the projects Physeco, MATH-AMSud, "Sistemas Dinámicos" funded by CSIC of Universidad de la República (Uruguay). The project leading to this publication has also received funding from Agencia Nacional de Investigación e Innovación (ANII) of Uruguay, Excellence Initiative of Aix-Marseille University - A*MIDEX and Excellence Laboratory Archimedes LabEx (ANR-11-LABX-0033), French "Investissements d'Avenir" programmes. 
Theorem 1. If $f$ is typical in $\mathcal{C}(M)$ (resp. $\mathcal{H}(M))$, then

$$
\overline{\mathcal{E}_{f}}=\overline{P e r_{f}}=\mathcal{O}_{f} .
$$

The following questions arise from Theorem 1, Are all the invariant measures in the closure of the ergodic measures? Are all the invariant measures pseudo-physical? We prove that the answer is negative for $C^{0}$-typical maps:

Theorem 2. If $f$ is typical in $\mathcal{C}(M)$ or if $f$ is typical in $\mathcal{H}(M)$, then the set $\mathcal{O}_{f}$ has empty interior in the set of all $f$-invariant measures.

In particular an open and dense set of $f$-invariant measures is not in the closure of the ergodic measures. Theorem 2 implies that the typical behaviour of homeomorphisms on manifolds widely differ from the typical behaviour of $C^{1}$ diffeomorphisms. In fact, Gelfert and Kwietniak proved that for $C^{1}$-typical diffeomorphisms the set of ergodic measures is dense in the space of invariant measures [GK, Theorem 8.1].

The proof of Theorem 1 is split into several pieces; its main components are Theorems 10, 20, 21 and Corollary 15. The main components of the proof of Theorem 2 are Theorems 5 and 12, Proposition 8, and Lemma 13.

The article is organized as follows. In Section 2 we give some background material and prove a technical lemma on the approximation of measures. In Section 3 we define the notion of shrinking sets, and we prove an extension of a result of Abdenur and Andersson, as well as some consequences of this result. We prove Theorem 2 in Section 4 . Finally, Sections 5 and 6 are dedicated to the proof of Theorem [1.

\section{THE SET-UP}

Let $M$ be a compact, connected, $C^{1}$ manifold of finite dimension $m \geq 1$, with or without boundary. Once a Riemannian structure is chosen on $M$, it defines a volume measure which we will refer to as the Lebesgue measure. It also defines a distance, $\operatorname{dist}(\cdot, \cdot)$, between points. We denote the $C^{0}$ distance on $C(M)$ by

$$
\rho(f, g):=\max _{x \in M} \operatorname{dist}(f(x), g(x)),
$$

This metric makes the set $C(M)$ a complete metric space and generates the $C^{0}$ topology on $C(M)$. Similarly, $\mathcal{H}(M)$ is a complete metric space with the $C^{0}$ topology generated by the distance

$$
\rho_{H}:=\max \left\{\rho(f, g), \rho\left(f^{-1}, g^{-1}\right)\right\} .
$$


The results of Theorems 1 and 2 hold independently of the choice of the Remannian structure on $M$. In fact, the topological and Borelmeasurable properties of the system depend on the metrizable topology of the manifold $M$, but not on the particular choice of its metric dist. The properties related to the pseudo-physical measures depend only on the set of pseudo-physical measures inside the space of all the Borel invariant measures. But this set is preserved if we substitute the previously chosen Lebesgue measure by any finite Borel measure equivalent to it (see Definition 3). Hence, the results remain unchanged if we change the choice of the volume form.

2.1. Pseudo-physical measures. For any point $x \in M$ and $f \in$ $C(M)$, let $p \omega(x)$ be the set of the Borel probability measures on $M$ that are the limits in the weak* topology of the convergent subsequences of the sequence

$$
\left\{\frac{1}{n} \sum_{j=0}^{n-1} \delta_{f^{j} x}\right\}_{n \in \mathbb{N}}
$$

where $\delta_{y}$ is the Dirac probability measure supported in $y \in M$.

A measure $\mu$ is called physical if the set of those $x \in M$ for which $p \omega(x)=\mu$ has positive Lebesgue measure. Note that we do not require physical measures to be ergodic.

In this article we consider a generalization of the above definition, introduced in [CE1] and studied in the $C^{1}$ case in [CE2, CCE1, CCE2]. We fix a distance, $d(\cdot, \cdot)$, in the space of probability measures that endows the weak* topology. It is easy to check that the following definition does not depend on the choice of this distance (see [CE1]).

Definition 3. A probability measure $\mu$ is called pseudo-physical if for all $\varepsilon>0$ the set $A_{\varepsilon}(\mu):=\{x \in M: d(p \omega(x), \mu)<\varepsilon\}$ has positive Lebesgue measure. We denote by $\mathcal{O}_{f}$ the set of pseudo-physical measures for $f$.

Note that $\mathcal{O}_{f}$ is always closed and non-empty, and that any pseudophysical measure is automatically $f$-invariant, and we do not require a pseudo-physical measure to be ergodic. (In [CE1, CE2, CCE1] pseudophysical measures were called SRB-like.)

2.2. The simplexes. We consider an atlas $\left\{\left(U_{\alpha}, \phi_{\alpha}\right)\right\}$ of the manifold $M$, where $U_{\alpha}$ are open sets whose union covers $M$, and $\phi_{\alpha}: U_{\alpha} \mapsto \mathbb{R}^{m}$ are $C^{1}$ diffeomorphisms.

An Euclidean $k$-simplex $(0 \leq k \leq m)$ is the convex hull of a finite set $\left\{x_{0}, x_{1}, \ldots, x_{k}\right\} \subset \mathbb{R}^{m}$ such that $\left\{x_{j}-x_{0}: 1 \leq j \leq k\right\}$ are linearly independent. 
A $k$-simplex of $M$ is a nonempty compact set $\bar{T}$ contained in a chart $\left(U_{\alpha}, \phi_{\alpha}\right)$ such that $\phi_{\alpha}(\bar{T})$ is an Eucliean $k$-simplex of $\mathbb{R}^{m}$. Throughout the article $T$ will denote the nonempty interior of an $m$-simplex $\bar{T}$.

$M$ is triangulable if there exits a finite family $\left\{\bar{T}_{1}, \ldots, \bar{T}_{\ell}\right\}$ of $m$ simplexes (called a triangulation) such that $T_{i} \cap T_{j}=\emptyset$ if $i \neq j$ and $\cup_{i} \bar{T}_{i}=M$.

It is well known that any compact $C^{1}$ manifold is triangulable [W], [M]. For any $\varepsilon>0$ there exists a triangulation of $M$ such that the diameters of all the simplexes $\bar{T}_{i}$ are smaller than $\varepsilon$. To prove this it suffices to notice that an Euclidean $m$-simplex can be decomposed into a finite number of $m$-simplexes of arbitrarily small diameter.

Consider a triangulation $\mathcal{T}:=\mathcal{T}_{m}:=\left\{\bar{T}_{1}^{m}, \ldots, \bar{T}_{h_{m}}^{m}\right\}$ of $M$. Define $\partial \mathcal{T}$ to be the union of the topological boundaries $\partial \bar{T}$ of the simplexes $\bar{T}$ of $\mathcal{T}$.

Consider an $m$-simplex $\bar{T}$ of $M$ and its associated chart $\left(U_{\alpha}, \phi_{\alpha}\right)$. Let $c$ be a point in the interior of $\bar{T}$, which we will call "centroid". Let $\left\{x_{0}, x_{1}, \ldots, x_{m}\right\} \subset \mathbb{R}^{m}$ be the vertices of $\phi_{\alpha}(\bar{T})$. Up to translation of the coordinates, it is not restrictive to assume that $\phi_{\alpha}(c)$ of $\phi_{\alpha}(\bar{T})$ is located at the origin. Suppose $\lambda>0$ is not too large. We denote $\lambda \bar{T} \subset U_{\alpha}$ the new simplex such that $\phi_{\alpha}(\lambda \bar{T})$ is the Euclidean $m$-simplex with vertices $\left\{\lambda x_{0}, \lambda x_{1}, \ldots, \lambda x_{m}\right\} \subset \phi_{\alpha}\left(\bar{U}_{\alpha}\right)$. Clearly, there exists a $\lambda_{0}>1$ (depending on $\bar{T}$ and the chosen centroid $c$ ) such that $\lambda \bar{T}$ is well defined for all $\lambda \in\left(0, \lambda_{0}\right)$.

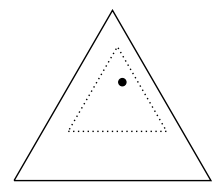

Figure 1. The simplex $\bar{T}$ is drawn with a solid line, while the simplex $\lambda \bar{T}$ for a $\lambda=1 / 2$ is drawn with a dotted line. The solid dot is the chosen centroid of the triangle located at the point $c \in T=\operatorname{int}(\bar{T}) \subset U_{\alpha}$.

2.3. A technical lemma. In the metrizable space of probability measures endowed with the weak* topology, consider the following distance between probabilities $\mu, \nu$ :

$$
d(\mu, \nu):=\sum_{i=1}^{\infty} \frac{1}{2^{i}}\left|\int \Psi_{i} d \mu-\int \Psi_{i} d \nu\right|,
$$

where $\left\{\Psi_{i}\right\}_{i=1}^{\infty}$ is a countable dense family in the space $\mathcal{C}^{0}(M,[0,1])$.

We will use the following lemma on the approximation of measures. 
Lemma 4. For any $\varepsilon>0$, there exists $q \geq 1$ such that, if $\mu$ and $\nu$ are probability measures of $M$ satisfying $\operatorname{supp}(\nu) \cup \operatorname{supp}(\mu) \subset \cup_{j=1}^{m} I_{j}$ for some pairwise disjoint connected closed sets, if $\operatorname{diam}\left(I_{j}\right) \leq 1 / q$, and if $\left|\nu\left(I_{j}\right)-\mu\left(I_{j}\right)\right| \leq 1 /$ qm for each $j$, then $d(\mu, \nu)<\varepsilon$.

Proof. Fix $n \geq 1$ such that $\sum_{i=n+1}^{+\infty} 2^{-i}<\varepsilon / 2$. Then, for any pair of probability measures $\mu, \nu$ we obtain

$$
d(\mu, \nu)<\frac{\varepsilon}{2}+\sum_{i=1}^{n} \frac{1}{2^{i}}\left|\int_{M} \Psi_{i} d \mu-\int_{M} \Psi_{i} d \nu\right| .
$$

The uniform continuity of the finite family of functions $\left\{\Psi_{i}\right\}_{1 \leq i \leq n}$, implies that there exists $\delta>0$ such that, if $\operatorname{dist}\left(x_{1}, x_{2}\right)<\delta$, then $\operatorname{dist}\left(\Psi_{i}\left(x_{1}\right), \Psi_{i}\left(x_{2}\right)\right)<\varepsilon / 4$ for all $1 \leq i \leq n$. Fix $q \in \mathbb{N}^{+}$such that $1 / q<\min (\delta, \varepsilon / 4)$.

The mean value theorem for integrals, yields for all $1 \leq i \leq n$

$$
\begin{gathered}
\int_{\bigcup_{j=1}^{m} I_{j}} \Psi_{i} d \mu=\sum_{j=1}^{m} \int_{I_{j}} \Psi_{i} d \mu=\sum_{j=1}^{m} \Psi_{i}\left(x_{j}\right) \mu\left(I_{j}\right) \text { for some } x_{j} \in I_{j}, \\
\int_{\bigcup_{j=1}^{m} I_{j}} \Psi_{i} d \nu=\sum_{j=1}^{m} \int_{I_{j}} \Psi_{i} d \nu=\sum_{j=1}^{m} \Psi_{i}\left(x_{j}^{\prime}\right) \nu\left(I_{j}\right) \text { for some } x_{j}^{\prime} \in I_{j} .
\end{gathered}
$$

From this we deduce

$$
\begin{gathered}
\int_{\bigcup_{j=1}^{m} I_{j}} \Psi_{i} d \mu-\int_{\bigcup_{j=1}^{m} I_{j}} \Psi_{i} d \nu=\sum_{j=1}^{m}\left(\Psi_{i}\left(x_{j}\right) \mu\left(I_{j}\right)-\Psi_{i}\left(x_{j}^{\prime}\right) \nu\left(I_{j}\right)\right) \\
=\sum_{j=1}^{m}\left(\Psi_{i}\left(x_{j}\right)\left(\mu\left(I_{j}\right)-\nu\left(I_{j}\right)\right)+\left(\Psi_{i}\left(x_{j}\right)-\Psi_{i}\left(x_{j}^{\prime}\right)\right) \nu\left(I_{j}\right)\right) .
\end{gathered}
$$

Since $\left|\nu\left(I_{j}\right)-\mu\left(I_{j}\right)\right| \leq 1 / q m$ for each $j$, we conclude

$$
\left|\int_{M} \Psi_{i} d \mu-\int_{M} \Psi_{i} d \nu\right| \leq \sum_{j=1}^{m} \frac{1}{q m}+\frac{\varepsilon}{4} \sum_{j=1}^{m} \nu\left(I_{j}\right)<\varepsilon / 2 \quad \forall 1 \leq i \leq n .
$$

Substituting this inequality in (1), finishes the proof of Lemma 4.

\section{SHRINKING SETS}

A periodic shrinking set of period $p \geq 1$, is a nonempty open set $I$ whose closure $\bar{I}$ is an $m$-simplex, such that $\left\{f^{j}(\bar{I})\right\}_{0 \leq j \leq p-1}$ is a family of pairwise disjoint sets, $f^{p}(\bar{I}) \subset I$, and $\operatorname{diam}\left(f^{j}(I)\right)<\operatorname{diam}(I)$ for all $1 \leq j \leq p-1$.

An nonempty open set $J$, whose closure $\bar{J}$ is an $m$-simplex, is eventually periodic shrinking, if there exists a periodic shrinking set $I$ and 
a transience time $n \in \mathbb{N}^{+}$such that $f^{n}(J) \subset I$, and $\operatorname{diam}\left(f^{j}(J)\right)<$ $\operatorname{diam}(J)$ for all $1 \leq j \leq n-1$.

Note that the same periodic or eventually periodic shrinking set for some $f \in \mathcal{C}(M)$ (resp. $\mathcal{H}(M)$ ) is also a periodic or eventually periodic shrinking set for all $g$ in a small neighborhood of $f$ in $\mathcal{C}(M)$ (resp. $\mathcal{H}(M))$.

If $I$ is a periodic or eventually periodic shrinking set with period $p$, then, by the Brouwer fixed point theorem, the map $f^{p}$ has a fixed point $x_{0} \in I$; hence, the point $x_{0}$ is periodic for $f$ with period $p$.

Theorem 5. For a typical map $f \in \mathcal{C}(M)$ (resp. $f \in \mathcal{H}(M)$ ), Lebesgue a.e. point $x \in M$ belongs to a sequence $\left\{I_{q}\right\}_{q \in \mathbb{N}^{+}}$of eventually periodic shrinking sets $I_{q}$ such that $\operatorname{diam}\left(I_{q}\right)<1 / q$.

Proof. For given natural numbers $q, k \in \mathbb{N}^{+}$, denote by $\mathcal{S}_{q, k}$ the set of maps in $\mathcal{C}(M)$ (resp. $\mathcal{H}(M)$ ), for which there exists a finite family of nonempty open sets, which we denote by $\left\{I_{1}, \ldots, I_{l}\right\}$, such that:

(i) $\operatorname{diam}\left(I_{i}\right)<1 / q$ for $i=1,2, \ldots, l$,

(ii) $I_{i}$ is a periodic or eventually periodic shrinking set,

(iii) $\operatorname{Leb}\left(M \backslash \bigcup_{i=1}^{l} I_{i}\right)<1 / k$.

The set $\mathcal{S}_{q, k}$ is open in $\mathcal{C}(M)$ (resp. $\mathcal{H}(M)$ ) since, for each $f \in \mathcal{S}_{q, k}$, the same family of such shrinking sets of $f$, is also a family of shrinking sets satisfying conditions (i), (ii), (iii) for any other map $g$ in a sufficiently small neighborhood of $f$.

Now, let us prove that $\mathcal{S}_{q, k}$ is dense in $\mathcal{C}(M)(\operatorname{resp} . \mathcal{H}(M))$. This will complete the proof of Theorem [5, since the set

$$
\mathcal{S}:=\bigcap_{q \geq 1} \bigcap_{k \geq 1} \mathcal{S}_{q, k}
$$

is a dense $G_{\delta}$-set, and by construction of $\mathcal{S}_{q, k}$, any map in $\mathcal{S}$ satisfies the conclusion of the Theorem.

To prove the denseness of $\mathcal{S}_{q, k}$ fix any map $f \in \mathcal{C}(M)(\operatorname{resp} . \mathcal{H}(M))$. For any $\varepsilon>0$, we will construct a perturbation $g \in \mathcal{S}_{q, k}$ such that $\rho(f, g)<\varepsilon\left(\right.$ resp. $\left.\rho_{H}<\varepsilon\right)$.

Choose $0<\delta<\min \{\varepsilon, 1 / q\}$ such that

$$
\operatorname{dist}(x, y)<\delta \Rightarrow \operatorname{dist}(f(x), f(y))<\frac{\varepsilon}{3},
$$

and if besides $f \in \mathcal{H}(M)$, then also $\operatorname{dist}\left(f^{-1}(x), f^{-1}(y)\right)<\frac{\varepsilon}{3}$. Consider a triangulation $\mathcal{T}:=\left\{\bar{T}_{1}, \ldots, \bar{T}_{l}\right\}$ of $M$ such that the diameters of all the simplexes $\bar{T}_{i}$ are smaller than $\delta$. For each $i$ we would like to choose a point $x_{i} \in T_{i}=\operatorname{int}\left(\bar{T}_{i}\right)$ such that $f\left(x_{i}\right) \in T_{j(i)}=\operatorname{int}\left(\bar{T}_{j(i)}\right)$ for some $j$ which depends on $x_{i}$. If $f$ is an homeomorphism the set of 
points in $T_{i}$ whose image is not in $\partial \mathcal{T}$ is a non-empty open set, and so we can choose such points $x_{i}$. If $f$ is merely continuous it is possible that such points do not exist. Then, we perturb $f$ to a continuous map $f_{1} \in \mathcal{C}(M)$ satisfying this property such that $\rho\left(f, f_{1}\right)<\varepsilon / 12$ and such that $\operatorname{dist}(x, y)<\delta \Rightarrow \operatorname{dist}\left(f_{1}(x), f_{1}(y)\right)<\varepsilon / 2$. For the sake of consistency of notation, if $f \in \mathcal{H}(M)$ or if $f \in \mathcal{C}(M)$ already satisfied this requirement we set $f_{1}:=f$.

Now we define "spherical coordinates" on each $\bar{T}_{i}$. To do this consider a chart $\left(U_{\alpha}, \phi_{\alpha}\right)$ such that $\bar{T}_{i} \subset U_{\alpha}$. Consider $S_{i}:=\phi_{\alpha}\left(\bar{T}_{i}\right)$. Up to a translation of the coordinates, it is not restrictive to assume that $0=\phi_{\alpha}\left(x_{i}\right)$. Recall that $x \in T_{i}=\operatorname{int}\left(\bar{T}_{i}\right)$; hence $0 \in \operatorname{int}\left(S_{i}\right)$. Therefore, there exists a small solid $m$-dimensional ball $B\left(0, r_{0}\right)$ lying inside $S_{i}$, and the spherical coordinate system $(r, \theta): r \in\left[0, r_{0}\right], \theta \in \mathbb{S}^{m}$ in it. Using the convexity of $S_{i}$ we can extend this coordinate system to all of $S_{i}$ by defining $R(\theta)$ to be the supremum of all $r$ such that the ray in the direction $\theta$ lies inside $S_{i}$ up to the distance $r$. For simplicity of notation, we normalize the $r$ coordinate, by setting $s:=r / R(\theta)$, thus $s \in[0,1]$ for all $\theta$. Finally we pull back these coordinates to $\bar{T}_{i}$ via the map $\phi_{\alpha}^{-1}$. For any $i$ and any $s_{0} \in(0,1]$ we will denote

$$
B_{S}^{i}:=\left\{(s, \theta) \in \bar{T}_{i}: s \in\left[0, s_{0}\right]\right\} .
$$

We consider an integer number $n \geq 1$ to be specified later. We define an homeomorphism $h$ of $M$ as follows. $h: \bar{T}_{i} \mapsto \bar{T}_{i}$ is given by by setting

$$
h(s, \theta):=\left(s^{n}, \theta\right) .
$$

Note that the map $h$ is uniquely defined on $\partial \mathcal{T}$, because for all $i$ the definition of $h$ on $\partial \bar{T}_{i}$ yields the identity map. Furthermore, note that since $h$ and $h^{-1}$ map each simplex $\bar{T}_{i}$ to itself, and the diameters of the $\bar{T}_{i}$ are at most $\delta$ we have

$$
\operatorname{dist}(x, h(x)) \leq \delta \quad \text { and } \quad \operatorname{dist}\left(x, h^{-1}(x)\right) \leq \delta .
$$

The map $g$ is then defined by

$$
g=f_{1} \circ h .
$$

We claim that, for an adequate choice of $n$, the map $g$ has the desired properties, i.e., $g \in \mathcal{S}_{q, k}$ and $\rho(g, f)<\varepsilon$ (and $\rho\left(g^{-1}, f^{-1}\right)<\varepsilon$ in the homeomorphism case).

Consider $y \in M$. Since $\operatorname{dist}(h(y), y)<\delta$ we have

$$
\operatorname{dist}\left(g(y), f_{1}(y)\right)=\operatorname{dist}\left(f_{1}(h(y)), f_{1}(y)\right)<\frac{\varepsilon}{2}
$$

and thus

$$
\rho(f, g)<\rho\left(f, f_{1}\right)+\rho\left(f_{1}, g\right)<\varepsilon .
$$


In the case that $f=f_{1} \in \mathcal{H}(M)$, we have $g^{-1}=h^{-1} \circ f_{1}^{-1}$. Consider $y \in M$, let $z=f^{-1}(y)=f_{1}^{-1}(y)$, then

$$
\operatorname{dist}\left(g^{-1}(y), f^{-1}(y)\right)=\operatorname{dist}\left(g^{-1}(y), f_{1}^{-1}(y)\right)=\operatorname{dist}\left(h^{-1}(z), z\right) \leq \delta<\varepsilon
$$

and thus

$$
\rho\left(g^{-1}, f^{-1}\right)<\varepsilon
$$

Now, to end the proof of Theorem 5 , it is enough to choose $n$ such that the map $g$ above constructed belongs to $\mathcal{S}_{q, k}$.

Choose $x_{i}$ as the centroid of $T_{i}$, choose $\lambda_{1} \in(0,1)$ close to 1 , and $\lambda_{2} \in\left(0, \lambda_{1}\right)$ close to 0 , and construct the simplexes $\lambda_{2} \bar{T}_{i} \subset \lambda_{1} \bar{T}_{i} \subset T_{i}=$ $\operatorname{int}\left(\bar{T}_{i}\right)$ whose interiors contain $x_{i}$. If $\lambda_{1}$ is close enough 1 , we have:

$$
\operatorname{Leb}\left(M \backslash\left(\cup_{i} \lambda_{1} \bar{T}_{i}\right)\right)<1 / k .
$$

Besides, if $\lambda_{1} \in(0,1)$ is close enough 1 , then $f_{1}\left(x_{i}\right) \in \operatorname{int}\left(\lambda_{1} \bar{T}_{j(i)}\right)$. So, if $\lambda_{2} \in\left(0, \lambda_{1}\right)$ is small enough, we have $f\left(x_{i}\right) \in f_{1}\left(\lambda_{2} \bar{T}_{i}\right) \subset \operatorname{int}\left(\lambda_{1} \bar{T}_{j(i)}\right)$ with $\operatorname{diam}\left(f_{1}\left(\lambda_{2} \bar{T}_{i}\right)\right)<\min _{j} \operatorname{diam}\left(\lambda_{1} \bar{T}_{j}\right)$ for all $i$.

We claim that there exists $n$ such that the sets $\lambda_{1} T_{i}$ are eventually periodic shrinking sets for $g$. In fact, choose a real number $s_{1}>0$ such that $\lambda_{1} \bar{T}_{i} \subset B_{s_{1}}^{i}$ and choose other real number $s_{2} \in\left(0, s_{1}\right)$ such that $B_{s_{2}}^{i} \subset \lambda_{2} \bar{T}_{i}$. Finally choose $n$ such that $s_{1}^{n}<s_{2}$. With these choices we have $h\left(B_{s_{1}}^{i}\right) \subset B_{s_{2}}^{i}$ and thus

$$
g\left(\lambda_{1} \bar{T}_{i}\right)=f \circ h\left(\lambda_{1} \bar{T}_{i}\right) \subset f \circ h\left(B_{s_{1}}^{i}\right) \subset f\left(B_{s_{2}}^{i}\right) \subset f\left(\lambda_{2} \bar{T}_{i}\right) \subset \lambda_{1} T_{j(i)} .
$$

Since there exists only a finite number $l$ of simplexes in the triangulation, this assertion implies that $\lambda_{1} T_{i}$ is an eventually periodic shrinking set with the sum of the transience time plus the period bounded by $l$. By construction, Properties (i), (ii) and (iii) are satisfied.

When $M$ is a manifold without boundary, the following result was proven by Abdenur and Andersson in $\mathrm{AA}$ for typical $f \in \mathcal{C}(M)$ in any finite dimension and for typical $f \in \mathcal{H}(M)$ in dimension at least two. Our proof works also when $M$ is a manifold with boundary, and also for $f \in \mathcal{H}(M)$ in dimension one.

Corollary 6. Let $f$ be a typical map in $C(M)($ resp. $\mathcal{H}(M))$. Then, for Lebesgue almost every point $x \in M$, the sequence

$$
\left\{\frac{1}{n} \sum_{j=0}^{n-1} \delta_{f^{j}(x)}\right\}_{n \in \mathbb{N}^{+}}
$$

of empirical probabilities is convergent, 
Proof. Consider the families $\mathcal{S}_{q, k}$ for $q, k \in \mathbb{N}^{+}$, defined at the beginning of the proof of Theorem 5. We proved that each $\mathcal{S}_{q, k}$ is open and dense, so typical $f$ belong to $\mathcal{S}:=\bigcap_{q, k \geq 1} \mathcal{S}_{q, k}$. Thus, it is enough that the assertion of this corollary holds for all $f \in \mathcal{S}$. Consider a continuous function $\phi: M \rightarrow \mathbb{R}$. Since $M$ is compact, for every $\varepsilon>0$ we can find a $\delta>0$ such that if $\operatorname{dist}(x, y)<\delta$ then $|\phi(x)-\phi(y)|<\varepsilon$. Suppose $f \in \mathcal{S}_{q, k}$ with $q$ such that $1 / q<\delta$. Due to Theorem 5, Lebesgue a.e. belongs to an eventually periodic shrinking set of diameter smaller than $1 / q$. It is enough to prove the convergence of the corollary for any iterate of $x$ instead of $x$; thus we can assume that $x \in I_{i}$ where $I_{i}$ is a periodic shrinking set. Denote the period of $I_{i}$ by $p$. Then for $n>p$ we write $n=\ell p+r$ with $0 \leq r<p$. Since $I_{i}$ is a periodic shrinking set with diameter smaller than $1 / q$ we have $\operatorname{dist}\left(f^{\ell p+r} x, f^{r} x\right) \leq \operatorname{diam} f^{r}\left(I_{i}\right) \leq$ $\operatorname{diam}\left(I_{i}\right)<1 / q<\delta$. Thus

$$
\left|\sum_{j=0}^{\ell p-1} \phi\left(f^{j} x\right)-\ell \sum_{j=0}^{p-1} \phi\left(f^{j} x\right)\right|<\ell \varepsilon
$$

Then

$$
\begin{aligned}
\mid \frac{1}{n} \sum_{j=0}^{n-1} \phi\left(f^{j} x\right) & -\frac{1}{p} \sum_{j=0}^{p-1} \phi\left(f^{j} x\right) \mid \leq \\
& \left|\frac{1}{n} \sum_{j=0}^{n-1} \phi\left(f^{j} x\right)-\frac{1}{\ell p} \sum_{j=0}^{\ell p-1} \phi\left(f^{j} x\right)\right|+ \\
& \left|\frac{1}{\ell p} \sum_{j=0}^{\ell p-1} \phi\left(f^{j} x\right)-\frac{1}{p} \sum_{j=0}^{\ell p-1} \phi\left(f^{j} x\right)\right| .
\end{aligned}
$$

Using (3) we see that second term is bounded by $\varepsilon / p<\varepsilon$. Using the triangle inequality once more we can bound the first term by

$$
\left|\frac{1}{n} \sum_{j=\ell p}^{n-1} \phi\left(f^{j} x\right)\right|+\left|\frac{r}{n \ell p} \sum_{j=0}^{\ell p-1} \phi\left(f^{j} x\right)\right| .
$$

Since $r$ is bounded, for $n$ sufficiently large this is bounded by $\varepsilon$, and the difference in (4) is bounded by $2 \varepsilon$.

We deduce that

$$
\limsup \frac{1}{n} \sum_{j=0}^{n-1} \phi\left(f^{j} x\right)-\liminf \frac{1}{n} \sum_{j=0}^{n-1} \phi\left(f^{j} x\right)<4 \varepsilon .
$$

Since $\varepsilon>0$ is arbitrary, the result follows. 
A map $f \in \mathcal{C}(M)$ is Lebesgue-a.e. strongly non positively expansive, if for any real number $\alpha>0$, and for Lebesgue a.e. $x \in M$,

$$
\operatorname{Leb}\left(\left\{y \in M: \operatorname{dist}\left(f^{n}(x), f^{n}(y)\right)<\alpha \quad \forall n \in \mathbb{N}\right\}\right)>0 .
$$

So, according to [AM, Definition 2.1], the Lebesgue measure is non positively expansive. Moreover, using the notation of [AM, Section 2]:

$$
\begin{gathered}
\operatorname{Leb}\left(\Phi_{\alpha}(x)\right) \neq 0 \text { Leb-a.e. } x \in M, \text { where } \\
\Phi_{\alpha}(x):=\left\{y \in M: \operatorname{dist}\left(f^{n}(y), f^{n}(x)\right)<\alpha .\right.
\end{gathered}
$$

Namely, the positive expansivity of the Lebesgue measure fails not only in some points, but Lebesgue a.e..

Corollary 7. Typical maps in $\mathcal{C}(M)$ (resp. $f \in \mathcal{H}(M)$ ) are Lebesguea.e. strongly non positively expansive.

Proof. Take any periodic or eventually periodic set $I$ with diameter smaller than $\alpha$. Then, $\operatorname{diam}\left(f^{j}(\bar{I})\right)<\alpha$ for all $j \geq 0$. Any two points $x, y \in I$ satisfy $\operatorname{dist}\left(f^{j}(x), f^{j}(y)\right)<\alpha$ for all $j \geq 0$. Thus for any point $x \in I$ we have

$$
\operatorname{Leb}\left(\left\{y \in M: \operatorname{dist}\left(f^{n}(x), f^{n}(y)\right)<\alpha \quad \forall n \in \mathbb{N}\right\}\right) \geq \operatorname{Leb}(I)>0 .
$$

Thus the result follows directly from Theorem 5 .

An $f \in \mathcal{C}(M)$ is called positively expansive if there exists a constant $\alpha>0$, called the expansivity constant, such that, for any two points $x, y \in M$, if $\operatorname{dist}\left(f^{n}(x), f^{n}(y)\right) \leq \alpha$ for all $n \in \mathbb{N}$, then $x=y$. Clearly a map which is Lebesgue a.e. strongly non positively expansive is not positively expansive. For compact connected manifolds with boundary it is known that every map is not positively expansive (Theorem 2.2.19, $[\mathrm{AH}]$ ). On the other hand, if $M$ has no boundary, then we have examples of positively expansive maps for example hyperbolic expanding toral diffeomorphisms.

3.1. The sets $A A$ and $A A_{1}$. For any $x \in M$ we define

$$
\mu_{x}:=\lim _{n \rightarrow \infty} \frac{1}{n} \sum_{j=0}^{n-1} \delta_{f^{j}(x)}
$$

when this limit exists; namely, when $p \omega(x)=\left\{\mu_{x}\right\}$. Let

$$
A A:=\left\{x \in M: p \omega(x)=\left\{\mu_{x}\right\}\right\} \text { and } A A_{1}:=\left\{x \in A A: \mu_{x} \in \mathcal{O}_{f}\right\} .
$$

For typical continuous maps or homeomorphisms, Corollary [ 6 states

$$
\operatorname{Leb}(A A)=\operatorname{Leb}(M),
$$


while CE2, Proposition 3.1 and Definition 3.2] implies that

$$
\operatorname{Leb}\left(A A_{1}\right)=\operatorname{Leb}(M) .
$$

Proposition 8. If $f$ is typical in $\mathcal{C}(M)$ (resp. $\mathcal{H}(M))$ then

$$
\mathcal{O}_{f}=\overline{\left\{\mu_{x}: x \in A A_{1}\right\}} \text {. }
$$

The set $\left\{x \in A A_{1}: d\left(\mu_{x}, \mu_{x_{0}}\right)<\varepsilon\right\}$ has positive Lebesgue measure for every $\varepsilon>0$ and for every $x_{0} \in A A_{1}$.

Proof. By definition of the set $A A_{1}$ we have $\left\{\mu_{x}: x \in A A_{1}\right\} \subset \mathcal{O}_{f}$. Besides, since $\mathcal{O}_{f}$ is closed in the weak* topology (see [CE1, Theorem 1.3]), we have $\overline{\left\{\mu_{x}: x \in A A_{1}\right\}} \subset \mathcal{O}_{f}$.

We turn to the other inclusion. Suppose $\mu \in \mathcal{O}_{f}$, i.e.,

$$
\operatorname{Leb}\{x \in M: d(p \omega(x), \mu)<\varepsilon)\}>0 \text { for all } \varepsilon>0 .
$$

Since $\operatorname{Leb}\left(A A_{1}\right)=1$ and $p \omega(x)=\mu_{x}$ for $x \in A A_{1}$, this is equivalent to

$$
\left.\operatorname{Leb}\left\{x \in A A_{1}: d\left(\mu_{x}, \mu\right)<\varepsilon\right)\right\}>0 \text { for all } \varepsilon>0 .
$$

Thus $\mu \in \overline{\left\{\mu_{x}: x \in A A_{1}\right\}}$. Since $\mu \in \mathcal{O}_{f}$ is arbitrary we conclude $\mathcal{O}_{f} \subset \overline{\left\{\mu_{x}: x \in A A_{1}\right\}}$. The first assertion of Proposition 8 is proven.

To prove the second assertion recall the definition of pseudo-physical measure, $\mu \in \mathcal{O}_{f}$ if the set $\{x \in M: d(p \omega(x), \mu)<\varepsilon\}$ has positive Lebesgue measure. Combining this with Equality (8) , we deduce that $\left\{x \in A A_{1}: d\left(\mu_{x}, \mu\right)<\varepsilon\right\}$ has positive Lebesgue measure, for any $\mu \in$ $\mathcal{O}_{f}$, in particular for $\mu_{x_{0}}$ for any $x_{0} \in A A_{1}$.

\subsection{Approximation of pseudo-physical measures by periodic}

measures. In this subsection we will prove Theorem 10, each pseudophysical measure can be arbitrarily approximated by atomic measures supported on periodic point. We will use the following lemma.

Lemma 9. Let $f \in \mathcal{C}(M)$ (resp. $f \in \mathcal{H}(M)$ ) and $\mu$ be an $f$-invariant measure. Assume that $I \subset M$ is a periodic shrinking set of period $p$, and denote $K:=\bigcup_{j=0}^{p-1} f^{j}(\bar{I})$. Then

$$
\mu\left(f^{j}(\bar{I})\right)=\mu(\bar{I})=\frac{\mu(K)}{p} \quad \forall j \geq 0 .
$$

If $\mu$ is additionally ergodic, then $\mu(K)$ is either zero or one.

Proof. For any $j \geq 0$ let $j=k p-i$ with $k \in \mathbb{N}^{+}$and $1 \leq i \leq p$. Using that $\mu$ is $f$-invariant, that $f^{p}(\bar{I}) \subset I$ and that $f^{-j}\left(f^{j}(\overline{\bar{I}})\right) \supset \bar{I}$ for all $j \geq 0$, we obtain:

$\mu\left(f^{j}(\bar{I})\right)=\mu\left(f^{-j}\left(f^{j}(\bar{I})\right) \geq \mu(\bar{I}) \geq \mu\left(f^{k p}(\bar{I})\right)=\mu\left(f^{-i}\left(f^{k p}(\bar{I})\right) \geq \mu\left(f^{j}(\bar{I})\right)\right.\right.$. 
Hence, all the inequalities above are equalities; and thus $\mu\left(f^{j}(\bar{I})\right)=$ $\mu(\bar{I})$ for all $j \geq 0$. Assertion (9) follows, since $\mu(K)=\sum_{j=0}^{p-1} \mu\left(f^{j}(\bar{I})\right)$.

Finally, since $f(K) \subset K$, we have $K \subset f^{-1}(f(K)) \subset f^{-1}(K)$. Since $\mu$ is ergodic, the set $K$ must have $\mu$-measure equal to zero or one.

Theorem 10. Typical maps $f \in \mathcal{C}(M)$ (resp. $f \in \mathcal{H}(M)$ ), satisfy $\mathcal{O}_{f} \subset \overline{\mathrm{Per}_{f}}$.

Proof. Choose $q_{n} \geq 1$ such that any two measures satisfying the $q_{n^{-}}$ approach conditions of Lemma 4, are mutually at distance smaller than $1 / n$. Let $\mu \in \mathcal{O}_{f}$. By the definition of pseudo-physical measure, the set $A_{n} \subset M$ of points $y$ such that

$$
d(p \omega(y), \mu)<1 / n,
$$

has positive Lebesgue measure. If $f$ is typical in $\mathcal{C}(M)$ or $\mathcal{H}(M)$, then Lebesgue a.e. $y_{n} \in A_{n}$ satisfy $p \omega\left(y_{n}\right)=\left\{\mu_{y_{n}}\right\}$ (Corollary 6) and is contained in an arbitrarily small eventually periodic shrinking set (Theorem 5). Therefore,

$$
d\left(\mu_{y_{n}}, \mu\right)<1 / n
$$

and $\mu_{y_{n}}$ is supported on $K_{n}:=\bigcup_{j=1}^{p_{n}} f^{j}\left(\bar{I}_{n}\right)$, where $I_{n}$ is a periodic shrinking set of period $p_{n}$, such that $\operatorname{diam}\left(I_{n}\right) \leq 1 / q_{n}$. Applying Lemma 9 and the definition of shrinking set yields $\mu_{y_{n}}\left(f^{j}\left(\bar{I}_{n}\right)\right)=1 / p_{n}$.

Since the set $I_{n}$ is shrinking periodic with period $p_{n}$, there exists at least one periodic orbit of period $p_{n}$ in $K_{n}$. Consider the periodic invariant measure $\nu_{n}$ supported on this periodic orbit. It also satisfies $\nu_{n}\left(f^{j}(\bar{I})\right)=1 / p_{n}$. So, applying Lemma 4 we deduce that

$$
\exists \nu_{n} \in \operatorname{Per}_{f}: d\left(\mu_{y_{n}}, \nu_{n}\right) \leq 1 / n .
$$

Combining Inequalities (10) and (11), we deduce that there exists sequence of periodic atomic measures $\nu_{n}$ such that $d\left(\nu_{n}, \mu\right)<2 / n$, finishing the proof of Theorem 10.

\section{Most InVARIAnt MEASURES ARE NOT PSEUDO-PHYSICAL}

In this section we will prove Theorem 2. We start with an extension of Theorem of Abdenur and Andersson in [AA, Theorem 3.6]:

Theorem 11. (Extension of Abdenur-Andersson Theorem) For typical maps in $C(M)$ there does not exist physical measures.

Proof. In [AA, Theorem 3.6], Abdenur and Andersson prove this assertion in the case that $M$ is a compact connected $C^{1}$ manifold without boundary. Now, let us prove that it also holds when $M$ has boundary. Given a manifold with boundary $M$, take two disjoint copies $M_{1}, M_{2}$ 
and identify each boundary point in $M_{1}$ with the same point in $M_{2}$. We obtain the double cover $D(M)$ of $M$ which is a $C^{1}$ manifold without boundary with the same dimension as $M$. See for example 9.32 in [L] for details of this consruction.

Suppose that $M$ has boundary, and denote by $\mathcal{V}$ the family of all the continuous map $f \in \mathcal{C}(M)$ such that $f(M) \subset M \backslash \partial M$. By construction $\mathcal{V}$ is open and dense in $\mathcal{C}(M)$. For each $f \in \mathcal{V}$, construct the nonempty set $\mathcal{S}_{f} \subset C(D(M))$ composed by all the continuous maps $g: D(M) \mapsto$ $D(M)$ such that $\left.g\right|_{M_{1}}=f$.

We claim that for any open set $\mathcal{U} \subset \mathcal{V}$ the set

$$
\mathcal{U}^{\prime}:=\bigcup_{f \in \mathcal{U}} \mathcal{S}_{f}=\left\{g \in C(D(M)):\left.g\right|_{M_{1}}=: f \in \mathcal{U}\right\}
$$

is open in $C(D(M))$; in particular $\mathcal{V}^{\prime}$ is open in $C(D(M))$. This claim follows from that fact that the restriction that sends each map $g \in C(D(M))$ to the map $\left.g\right|_{M_{1}}: M_{1} \mapsto g\left(M_{1}\right) \subset M$ is a continuous operator. Therefore, the preimage $\mathcal{U}^{\prime}$ by that restriction, of the open family $\mathcal{U} \subset \mathcal{V} \subset C(M) \subset C\left(M_{1}, D(M)\right)$, is open.

Next we claim that a converse-like statement is true: for any open set $\mathcal{U}^{\prime} \subset \mathcal{V}^{\prime} \subset C(D(M))$ the set

$$
\mathcal{U}:=\left\{f \in \mathcal{V}: \exists g \in \mathcal{U}^{\prime} \subset C(D(M)) \text { such that }\left.g\right|_{M_{1}}=f\right\}
$$

is open in $C(M)$. In fact, since $\mathcal{U}^{\prime} \subset \mathcal{V}^{\prime}$, the restriction $\left.g\right|_{M_{1}}$ for each $g \in \mathcal{U}^{\prime}$, belongs to $\mathcal{V}$. Besides, the restriction that sends each map $g \in \mathcal{V}^{\prime}$ to the map $f:=\left.g\right|_{M_{1}} \in \mathcal{V}$ is an open operator. Therefore, the image $\mathcal{U}$ by that restriction, of the open family $\mathcal{U}^{\prime}$, is open.

Since Theorem 11 holds for typical maps $g \in C(D(M))$, and $\mathcal{V}^{\prime}$ is nonempty and open in $C(D(M))$, it also holds for typical maps $g \in \mathcal{V}^{\prime}$. Namely, there exists a sequence $\left\{\mathcal{U}_{n}^{\prime}\right\}_{n \geq 1}$ of open and dense subsets of $\mathcal{V}^{\prime}$ such that assertions a) and b) hold for all $g \in \bigcap_{n \geq 1} \mathcal{U}_{n}^{\prime} \subset \mathcal{V}^{\prime}$. Consider the preimages $\mathcal{U}_{n} \subset \mathcal{V}$ defined in (12), of the sets $\mathcal{U}_{n}$. Since $\mathcal{U}_{n}$ is open and dense in $\mathcal{V}^{\prime}$, the above assertions imply that $\mathcal{U}_{n}$ is open and dense in $\mathcal{V}$; hence also in $\mathcal{C}(M)$.

If Theorem 11 holds for a map $g \in \mathcal{V}^{\prime} \subset C(D(M))$, then it also holds for the restriction $\left.g\right|_{M_{1}} \in \mathcal{V} \subset \mathcal{C}(M)$. We conclude that it holds for the countable intersection $\bigcap_{n \geq 1} \mathcal{U}_{n}$ of open and dense sets in $\mathcal{C}(M)$. In other words, Theorem 11 holds for typical maps in $\mathcal{C}(M)$.

The following theorem follows easily from results of Hurley and his coauthors on the existence of many periodic points for homeomorphism of manifolds of dimension at least two without boundary AHK, H1. 
We give a different proof which also works for manifolds with boundary and also in dimension one.

Theorem 12. Typical maps in $\mathcal{C}(M)$ (resp. $\mathcal{H}(M)$ ) are not uniquely ergodic.

Proof. First, let us argue in $\mathcal{C}(M)$. Recall that Lebesgue a.e. $x \in M$ belongs to the set $A A_{1}$ defined by Equality (6). We claim that if $f \in \mathcal{C}(M)$ is typical, then for any $\varepsilon>0$ and any $x_{0} \in A A_{1}$ the set $\left\{x \in A A_{1}: \mu_{x} \neq \mu_{x_{0}}\right.$ and $\left.d\left(\mu_{x}, \mu_{x_{0}}\right)<\varepsilon\right\}$ has positive Lebesgue measure.

To prove the claim we argue by contradiction, assume that there exists $x_{0} \in A A_{1}$ and $\varepsilon>0$ such that

$$
\operatorname{Leb}\left\{x \in A A_{1}: \mu_{x} \neq \mu_{x_{0}} \text { and } d\left(\mu_{x_{0}}, \mu_{x}\right)<\varepsilon\right\}=0 .
$$

Since $\mu_{x_{0}}$ is pseudo-physical, by definition we have

$$
\operatorname{Leb}\left(\left\{x \in M: d\left(p \omega(x), \mu_{x_{0}}\right)<\varepsilon\right\}\right)>0 .
$$

Since $\operatorname{Leb}\left(A A_{1}\right)=1$ we deduce that

$$
\operatorname{Leb}\left(\left\{x \in A A_{1}: d\left(\mu_{x}, \mu_{x_{0}}\right)<\varepsilon\right\}\right)>0 .
$$

Combining this last assertion with Inequality (13), we obtain that

$$
\left.\operatorname{Leb}\left(\left\{x \in A A_{1}: \mu_{x}=\mu_{x_{0}}\right)\right\}\right)>0 .
$$

So, $\mu_{x_{0}}$ is a physical measure, contradicting Theorem 11, the claim is proven.

Since the measures $\mu_{x}$ are $f$-invariant for all $x \in A A_{1}$, we deduce from the claim that $f$ is not uniquely ergodic.

Now let us prove Theorem 12 for typical $f \in \mathcal{H}(M)$. From Theorem 5, there exists a periodic shrinking set $I$ with arbitrarily small diameter. Denote by $p$ the period of $I$, and by $x_{0} \in I$ a periodic point of period $p$. Recall that $\operatorname{diam}\left(f^{j}(I)\right)<\operatorname{diam}(I)$. Besides the sets $f^{i}(\bar{I})$ are pairwise disjoint for $j \in\{0, \ldots, p-1\}, f^{p}(\bar{I}) \subset I$, and this inclusion is strict since $\operatorname{diam}\left(f^{p}(I)\right)<\operatorname{diam}(I)$. Therefore, if $\operatorname{diam}(I)$ is small enough, then

$$
K:=\bigcup_{j=1}^{p} f^{j}(\bar{I}) \neq M .
$$

Denote by $\nu$ the atomic invariant measure supported on the periodic orbit of $x_{0}$. We clain that there exists $\delta>0$ such that for any Borel probability measure $\mu$ (not necessarily invariant), if $\operatorname{dist}(\mu, \nu)<\delta$, then $\mu(I)>0$. 
To prove the claim, we argue by contradiction. Assume that there exists $\mu_{n} \rightarrow \nu$ such that $\mu_{n}(I)=0$. Consider $\psi: M \mapsto[0,1]$ defined by

$$
\psi(x):=\frac{\operatorname{dist}(x, M \backslash I)}{\operatorname{dist}\left(x, f^{p}(\bar{I})\right)+\operatorname{dist}(x, M \backslash I)} .
$$

This function is continuous and satisfies $\left.\psi\right|_{f^{p}(\bar{I})}=1,0<\psi(x)<1$ if $\left.x \in I \backslash f^{p}(\bar{I})\right)$ and $\psi(x)=0$ if $x \notin I$. Since $\mu_{n}(I)=0$, we obtain $\int \psi d \mu_{n}=0$, and taking the weak*-limit of $\mu_{n}$, we deduce $\int \psi d \nu=0$; hence $\psi=0 \nu$-a.e., which contradicts that $\nu(I)=1 / p>0$ and $\psi>0$ for all $x \in I$, finishing the proof of the claim.

Now, let us prove that $f$ is not uniquely ergodic. Arguing by contradiction, if $\nu$ were the unique invariant measure, then $(1 / n) \sum_{j=0}^{n-1} \delta_{f^{j}(x)}$ would converge uniformly to $\nu$ for all $x \in M$ as $n \rightarrow+\infty$. Therefore, there would exist $n_{0} \geq 1$ such that

$$
\operatorname{dist}\left(\nu,\left(1 / n_{0}\right) \sum_{j=0}^{n_{0}-1} \delta_{f^{j}(x)}\right)<\delta \quad \forall x \in M .
$$

Applying the claim for all $x \in M$, there would exist $0 \leq j=j(x) \leq$ $n_{0}-1$ such that $f^{j}(x) \in I$. Since $I$ is shrinking periodic, we would deduce that $f^{n_{0}}(x) \in K$ for all $x \in M$. Therefore, applying assertion (14), the image of $M$ by the homeomorphism $f^{n_{0}}: M \mapsto M$ would not be $M$, which is a contradiction.

Now, we state the main lemma to be used to prove Theorem 2.

Lemma 13. Suppose that $f$ is a typical map in $\mathcal{C}(M)$ (resp. $\mathcal{H}(M)), \mu_{1}$ is $f$-invariant and supported on $K=\bigcup_{j=0}^{p-1} f^{j}(\bar{I})$, where $I$ is a periodic shrinking set of period $p$, and that $\mu_{2}$ is $f$-invariant such that $\mu_{2}(K)=$ 0 . Then, no convex combination $\nu=\lambda \mu_{1}+(1-\lambda) \mu_{2}$ with $0<\lambda<1$ is pseudo-physical.

Proof. Since $I$ is a shrinking periodic set with period $p$, it is is open, $\bar{I}$ is an $m$-simplex, and $f^{p}(\bar{I}) \subset I$. Consider the continuous real function $\psi: M \rightarrow[0,1]$ defined by Equality (14). It satisfies $\left.\psi\right|_{f^{p}(\bar{I})}=1$, $0<\psi(x)<1$ if $x \in I \backslash f^{p}(\bar{I})$, and $\psi(x)=0$ if $x \notin I$.

Since $\mu_{1}$ is supported on $K=\bigcup_{j=0}^{p-1} f^{j}(\bar{I})$, we can apply Lemma 9 to deduce that

$$
\mu_{1}(\bar{I})=\mu_{1}\left(f^{p}(\bar{I})\right)=\frac{1}{p} ; \quad \text { hence } \mu_{1}\left(I \backslash f^{p}(\bar{I})\right)=0 .
$$

We obtain $\int \psi d \mu_{1}=\mu_{1}\left(f^{p}(\bar{I})\right)=1 / p$ and $\int \psi d \mu_{2}=\mu_{2}(I)=0$. Therefore $0<\int \psi d \nu=\lambda / p<1 / p$. 
Choose $\varepsilon>0$ such that for any measure $\mu$, if $d(\nu, \mu)<\varepsilon$, then $\int \psi d \mu>0$ and $\int \psi d \mu<1 / p$.

Consider the set

$$
A_{\varepsilon}^{\prime}(\nu):=\left\{x \in A A: \operatorname{dist}\left(\mu_{x}, \nu\right)<\varepsilon\right\},
$$

where the set $A A$ is defined in Equality (6). We claim that the set $A_{\varepsilon}^{\prime}(\nu)$ is empty. Arguing by contradiction, assume that there exists $x \in A_{\varepsilon}^{\prime}(\nu)$. Then, from the choice of $\varepsilon$, we have

$$
0<\int \psi d \mu_{x}<1 / p
$$

and from (5) we deduce that there exists $n_{0} \geq 1$ such that

$$
\frac{1}{n} \sum_{j=0}^{n-1} \psi\left(f^{j}(x)\right)=\int \psi\left(\frac{1}{n} \sum_{j=0}^{n-1} \delta_{f^{j}(x)}\right)>0 \forall n \geq n_{0} .
$$

Thus, there exists $n_{1} \geq 1$ such that $\psi\left(f^{n_{1}}(x)\right)>0$; hence $f^{n_{1}}(x) \in I$. This implies that the future orbit of $f^{n_{1}} x$ is contained in $K$. Hence, $\mu_{x}$ is supported on $K$. Since $\mu_{x}$ is $f$-invariant, we apply Lemma 9 to deduce that $\mu_{x}\left(f^{p}(\bar{I})\right)=1 / p$ and $\mu_{x}\left(I \backslash f^{p}(\bar{I})\right)=0$. Therefore,

$$
\int \psi d \mu_{x}=\mu_{x}\left(f^{p}(\bar{I})\right)=\frac{1}{p}
$$

contradicting (15). We have proved that $A_{\varepsilon}^{\prime}(\nu)$ is empty.

The definition of pseudo-physical measure $\mu$ asserts that

$$
\operatorname{Leb}\left(A_{\varepsilon}(\mu)\right)>0 \quad \forall \varepsilon>0 .
$$

But (17) implies that $\operatorname{Leb}\left(A_{\varepsilon}^{\prime}(\nu)\right)=\operatorname{Leb}\left(A_{\varepsilon}(\nu)\right)$, which equals zero because $A_{\varepsilon}^{\prime}(\nu)=\emptyset$. We conclude that $\nu$ is not pseudo-physical.

\subsection{End of the proof of Theorem 2 .}

Proof. From [CE1, Theorem 1.3] the set $\mathcal{O}_{f}$ is closed. Let us prove that its interior in $\mathcal{M}_{f}$ is empty. Fix $\mu \in \mathcal{O}_{f}$. Since a typical map is not uniquely ergodic (recall Theorem 12), there exists an ergodic measure $\nu \neq \mu$. Denote by $\varepsilon:=d\left(\mu_{x}, \nu\right)>0$. Consider an arbitrary $\delta \in(0, \varepsilon / 2)$.

From Proposition 8 , we can find $x_{0} \in A A_{1}$ such that $d\left(\mu_{x_{0}}, \mu\right)<\delta$. So, to prove that $\mu$ does not belong to the interior of $\mathcal{O}_{f}$ in $\mathcal{M}_{f}$, it is enough to prove that the $\delta$-neighborhood of $\mu_{x_{0}}$ in $\mathcal{M}_{f}$ is not contained in $\mathcal{O}_{f}$. To do that, it is enough to find a sequence $\left\{\mu_{n}\right\}_{n} \subset \mathcal{M}_{f}$ converging to $\mu_{x_{0}}$ and such that $\mu_{n} \notin \mathcal{O}_{f}$.

Applying Theorem [5 and Equality (8), and taking into account the second part of Proposition 8, the point $x_{0}$ could be chosen to belong to arbitrarily small eventually periodic shrinking sets. Therefore, for any fixed $q \in \mathbb{N}^{+}$(that will be chosen later), the probability measure 
$\mu_{x_{0}}$ is supported on the orbit of a periodic shrinking set $I_{q}$ of diameter smaller than $1 / q$. Denote by $p$ the period of $I_{q}$, and denote

$$
K=\bigcup_{j=0}^{p-1} f^{j}\left(\bar{I}_{q}\right)
$$

We have $\mu_{x_{0}}(K)=1$. So, applying Lemma 9 we deduce

$$
\mu_{x_{0}}\left(f^{j}(\bar{I})\right)=1 / p \quad 0 \leq j \leq p-1 .
$$

Applying Lemma 4, construct $q \geq 1$ such that if $\mu$ is a probability measure satisfying $\mu\left(f^{j}(\bar{I})\right)=\mu_{x}\left(f^{j}(\bar{I})\right)=1 / p$ with $\operatorname{diam}\left(f^{j}(\bar{I})\right)<1 / q$ for all $0 \leq j \leq p-1$, then $d\left(\mu, \mu_{x}\right)<\delta$. Since $d(\mu, \nu)=\varepsilon>\delta$, we deduce that $\nu\left(f^{j}(\bar{I})\right) \neq 1 / p$ for some $j$. But applying Lemma 9 $\nu\left(f^{j}(\bar{I})\right)=\nu(K) / p$ for all $j$, with $\nu(K) \in\{0,1\}$. Since $\nu$ is ergodic, we conclude that $\nu(K)=0$. Therefore, applying Lemma 13, $\mu_{n}:=$ $\lambda_{n} \mu_{x}+\left(1-\lambda_{n}\right) \nu \in \mathcal{M}_{f} \backslash \mathcal{O}_{f}$ for all $0<\lambda_{n}<1$. Taking $\lambda_{n} \rightarrow 1^{-}$, we obtain $\mu_{n} \rightarrow \mu_{x}$, with $\mu_{n} \in \mathcal{M}_{f} \backslash \mathcal{O}_{f}$, as wanted.

5. PSEudo-Physical, ERGodic ANd PERIOdiC MEASURES.

A $\delta$-pseudo-orbit of $f$ is a sequence $\left\{y_{n}\right\}_{n \in \mathbb{N}} \subset M$ such that

$$
\operatorname{dist}\left(f\left(y_{n}\right), y_{n+1}\right)<\delta \quad \forall n \in \mathbb{N} \text {. }
$$

A $\delta$-pseudo-orbit $\left\{y_{n}\right\}_{n \in \mathbb{N}}$ is periodic with period $p \geq 1$, if

$$
y_{n+p}=y_{n} \quad \forall n \in \mathbb{N} \text {. }
$$

A map $f \in \mathcal{C}(M)$ has the periodic shadowing property if for all $\varepsilon>0$, there exists $\delta>0$ such that, if $\left\{y_{n}\right\}_{n \in \mathbb{N}}$ is any periodic $\delta$-pseudo-orbit, then, at least one periodic orbit $\left\{f^{n}(x)\right\}_{n \in \mathbb{N}}$ satisfies

$$
\operatorname{dist}\left(f^{n}(x), y_{n}\right)<\varepsilon \quad \forall n \in \mathbb{N} .
$$

We will also use the following result, which is the accumulation of many authors' work.

Theorem 14. Typical maps $f \in \mathcal{C}(M)$ (resp. $\mathcal{H}(M)$ ) have the periodic shadowing property.

Proof. The statement follows from [KMOP, Theorem 1.2] and [PP, Theorem 1]. Earlier special cases where treated in [Y] and [O].

Recall that $\mathcal{E}_{f}$ denotes the set of ergodic measures, and $\mathrm{Per}_{f}$ denotes the set of invariant measures supported on periodic orbits of $f$. As a consequence of Theorem 14:

Corollary 15. For a typical map $f \in \mathcal{C}(M)$ (resp. $f \in \mathcal{H}(M)$ ) we have $\mathcal{E}_{f} \subset \overline{\operatorname{Per}_{f}}$. 
Remark. The closing lemma and the closeability properties (see for instance [CS, Definition 2.1] and [GK, Definitions 4.1 and 4.5]), state that for all $\epsilon>0$ there exists $\delta>0$ such that, for a finite piece of orbit $\left\{f^{j}(y\}_{0 \leq j \leq p}\right.$ verifying $\operatorname{dist}\left(f^{p}(y), y\right)<\delta$, there exists a periodic point $x$, whose period is $p$ or at least $(\epsilon \cdot p)$ - near $p$, satisfying $\operatorname{dist}\left(f^{j}(y), f^{j}(x)\right)<$ $\epsilon$ for all $0 \leq j \leq p$. It is known that the closing lemma and the closeability properties imply $\mathcal{E}_{f} \subset \overline{\operatorname{Per}_{f}}$ (see for instance CS, Lemma 2.2] and [GK, Theorems 4.10]). Nevertheless, we do not know if typical maps in $C^{0}(M)$ satisfy the closing lemma or the closeability properties.

Proof of Corollary 15. From the definition of distance in the weak* topology of the space of probability measures it is standard to check that for all $\varepsilon_{0}>0$, there exists $\varepsilon>0$, such that, for any two points $x_{1}, x_{2} \in M$,

$$
\operatorname{dist}\left(x_{1}, x_{2}\right)<\varepsilon \Rightarrow d\left(\delta_{x_{1}}, \delta_{x_{2}}\right)<\varepsilon_{0} .
$$

Fix any $\mu \in \mathcal{E}_{f}$. Since $\mu$ is ergodic, we have $p \omega(x)=\{\mu\}$ for $\mu$-a.e. $x \in$ $M$. Fix such a point $x$; then there exists $n_{0} \geq 1$ such that

$$
d\left(\frac{1}{n} \sum_{j=0}^{n-1} \delta_{f^{j}(x)}, \mu\right)<\varepsilon_{0} \quad \forall n \geq n_{0} .
$$

Given $\varepsilon$, choose $\delta>0$ given by Theorem 14, By Poincaré Recurrence Lemma, the point $x$ can be chosen to be recurrent. Thus

$$
d\left(f^{p-1}(x), x\right)<\delta \quad \text { for some } p \geq n_{0} .
$$

Construct the periodic $\delta$-pseudo-orbit $\left\{y_{n}\right\}_{n \in \mathbb{N}}$ of period $p$ defined by $y_{n}=f^{n}(x)$ for all $0 \leq n<p, y_{n+p}=y_{n}$ for all $n \geq 0$. Applying Theorem 14, there exists a periodic orbit $\left\{f^{n}(z)\right\}_{n \geq 0}$, such that $\operatorname{dist}\left(f^{n}(z), y_{n}\right)<\varepsilon \forall n \geq 0$. By construction, if $i p \leq n<(i+1) p$ and $i \geq 0$ then $\operatorname{dist}\left(f^{n}(z), f^{n-i p}(x)\right)<\varepsilon$. Thus, from the choice of $\varepsilon$, we obtain $d\left(\delta_{f^{n}(z)}, \delta_{f^{n-i p}(x)}\right)<\varepsilon_{0}$ Denote by $q$ the period of $z$. Taking into account that balls are convex in the weak*-distance in the space of probabilities, we deduce

$$
d\left(\frac{1}{q p} \sum_{j=0}^{q p-1} \delta_{f^{j}(z)}, \quad \frac{1}{q p} q \cdot \sum_{j=0}^{p-1} \delta_{f^{j}(x)}\right)<\varepsilon_{0} .
$$

For the atomic invariant measure $\nu$ supported on the periodic orbit of $z$, we have

$$
\nu=\frac{1}{q} \sum_{j=0}^{q-1} \delta_{f^{j}(z)}=\frac{1}{q p} \sum_{j=0}^{q p-1} \delta_{f^{j}(z)} .
$$


Thus,

$$
d\left(\nu, \frac{1}{p} \sum_{j=0}^{p-1} \delta_{f^{j}(x)}\right)<\varepsilon_{0} .
$$

Together with (16), this implies that for any $\varepsilon_{0}$ the given ergodic measure $\mu$ is $2 \varepsilon_{0}$-approximated by some measure $\nu \in \operatorname{Per}_{f}$, finishing the proof of Corollary 15.

An invariant measure $\mu$ is called infinitely shrinked if there exists a sequence $\left\{I_{q}\right\}_{q \geq 0}$ of periodic shrinking intervals $I_{q}$, of periods $p_{q}$, such that $\operatorname{diam}\left(I_{q}\right)<1 / q$ and $\mu\left(\bigcup_{i=1}^{p_{q}} f^{j}\left(\bar{I}_{q}\right)\right)=1$ for all $q \geq 1$. We denote by $\operatorname{Shr}_{f} \subset \mathcal{M}_{f}$ the set of infinitely shrinked invariant measures. Define

$$
A A_{2}:=\left\{x \in A A_{1}: \quad \mu_{x} \in \operatorname{Shr}_{f}\right\} .
$$

Theorem 16. For a typical map $f \in \mathcal{C}(M)$ (resp. $f \in \mathcal{H}(M)$ ),

$$
\operatorname{Leb}\left(A A_{2}\right)=1 \quad \text { and } \quad \mathcal{O}_{f}=\overline{\left\{\mu_{x}: x \in A A_{2}\right\}}=\overline{\operatorname{Shr}_{f}} \text {. }
$$

Proof. From Theorem 5, Lebesgue-a.e. $x \in M$ belongs to a sequence of eventually periodic or periodic shrinking sets $J_{q}$ with $\operatorname{diam}\left(J_{q}\right)<1 / q$. Every eventually periodic shrinking set $J_{q}$ wanders under $f$ until it drops into a periodic shrinking set $I_{q}$ with $\operatorname{diam}\left(I_{q}\right)<\operatorname{diam}\left(J_{q}\right)<1 / q$. By the definition of periodic shrinking set, every point of $I_{q}$ has all the measures of $p \omega(x)$ supported on the compact set

$$
K_{x, q}:=\bigcup_{j=0}^{p_{q}-1} f^{j}\left(\bar{I}_{q}\right) .
$$

In particular for Lebesgue almost all $x \in A A_{1}$, the limit measure $\mu_{x}$ defined by (5), is supported on $K_{x, q}$. Thus, for a.e. $x \in A A_{1}$ we have $\mu_{x} \in \operatorname{Shr}_{f}$. Taking into account (8), the above assertion implies that the set $A A_{2}$ has full Lebesgue measure.

By construction, $A A_{2} \subset A A_{1}$. So, applying Proposition 8 , we obtain:

$$
\overline{\left\{\mu_{x}: x \in A A_{2}\right\}} \subset \overline{\left\{\mu_{x}: x \in A A_{1}\right\}}=\mathcal{O}_{f} .
$$

To obtain the opposite inclusion, we apply [CE1, Theorem 1.5]): $\mathcal{O}_{f}$ is the minimal weak*-compact set of probability measures, that contains $p \omega(x)$ for Lebesgue a.e. $x$. Since $\overline{\left\{\mu_{x}: x \in A A_{2}\right\}}$ is weak*-compact and contains $p \omega_{x}=\left\{\mu_{x}\right\}$ for Lebesgue almost all $x$ (because $\operatorname{Leb}\left(A A_{2}\right)=$ $\operatorname{Leb}(M))$, we conclude that

$$
\overline{\left\{\mu_{x}: x \in A A_{2}\right\}} \supset \mathcal{O}_{f} .
$$

The inclusion $\overline{\left\{\mu_{x}: x \in A A_{2}\right\}} \subset \overline{\mathrm{Shr}_{f}}$ follows trivially from the definition of the set $A A_{2}$. Now, let us prove the opposite inclusion. We will 
prove that every shrinking measure is pseudo-physical. Let $\mu \in \mathrm{Shr}_{f}$. For any $\varepsilon>0$, choose $q \geq 1$ as in Lemma 4. By the definition of shrinking measure, there exists a periodic shrinking set $I_{q^{\prime}}^{\prime}$ such that $\mu$ is supported on

$$
K_{\mu}=\bigcup_{j=0}^{p_{q^{\prime}}-1} f^{j}\left(\overline{I_{q^{\prime}}^{\prime}}\right)
$$

for the periodic shrinking set $I_{q^{\prime}}^{\prime}$ of period $p_{q^{\prime}}$, such that $\operatorname{diam}\left(\overline{I_{q^{\prime}}^{\prime}}\right)<$ $1 / q^{\prime}$; hence $\operatorname{diam}\left(f^{j}\left(\bar{I}_{q^{\prime}}^{\prime}\right)\right)<1 / q^{\prime} \forall 1 \leq j \leq p_{q^{\prime}}, \mu\left(K_{\mu}\right)=1$.

Besides, for any point $x \in I_{q^{\prime}}^{\prime}$, any measure in $p \omega_{x}$ is also supported on $K_{\mu}$. If additionally $x \in A A_{1}$, then $p \omega_{x}=\left\{\mu_{x}\right\}$, so $\mu_{x}\left(K_{\mu}\right)=1$. Finally, applying Lemmas 4 and 9, we deduce that the measures $\mu \in$ $\mathrm{Shr}_{f}$ and $\mu_{x}$ given above satisfy

$$
d\left(\mu, \mu_{x}\right)<\varepsilon \text { for any } x \in I_{q} \cap A A_{1} .
$$

Since $\operatorname{Leb}\left(I_{q^{\prime}}^{\prime} \cap A A_{1}\right)=\operatorname{Leb}\left(I_{q^{\prime}}^{\prime}\right)>0$, the basin $A_{\varepsilon}(\mu)$ has positive Lebesgue measure; namely $\mu$ is pseudo-physical.

We have shown that every shrinking measure is pseudo-physical. Since the set $\mathcal{O}_{f}$ of pseudo-physical measures is closed, we conclude

$$
\overline{\operatorname{Shr}_{f}} \subset \mathcal{O}_{f},
$$

finishing the proof of Theorem 16 .

Theorem 17. For any map $f \in \mathcal{C}(M)$ (resp. $f \in \mathcal{H}(M)$ ), if $\mu \in S h r_{f}$, then it is ergodic.

Before proving Theorem 17 let us deduce its main consequence:

Corollary 18. For a typical map $f \in \mathcal{C}(M)$ (resp. $f \in \mathcal{H}(M))$ :

$$
\mathcal{O}_{f}=\overline{S h r_{f}} \subset \overline{\mathcal{E}_{f}}=\overline{P e r}_{f} .
$$

The corollary immediately follows by combining Corollary 15 with Theorems 16 and 17. At the end of the next section we will prove that for typical maps these sets are all equal.

Proof of Theorem 17. Fix $f \in \mathcal{C}(M)$. Suppose $\mu \in \mathrm{Shr}_{f}$, and $\mu_{1}, \mu_{2} \in$ $\mathcal{M}_{f}$ such that

$$
\mu=\lambda \mu_{1}+(1-\lambda) \mu_{2}, \quad \text { with } 0<\lambda<1,
$$

We shall prove that $\mu_{1}=\mu_{2}=\mu$; namely $\mu$ is extremal in the convex compact set of invariant measures; hence ergodic.

Take arbitrary $\varepsilon>0$ and fix $q \geq 1$ as in Lemma 4. By the definition of infinitely shrinking measures, there exists a periodic shrinking set 
$I_{q}$, with $\operatorname{diam}\left(I_{q}\right)<1 / q$, and period $p_{q}$, whose $f$-orbit $K_{q}$ supports $\mu$. The definition of periodic shrinking set and Lemma 9 tell us:

$$
\mu\left(f^{j}\left(\bar{I}_{q}\right)\right)=\frac{1}{p_{q}}, \quad \operatorname{diam}\left(f^{j}\left(\bar{I}_{q}\right)\right)<1 / q \quad \forall 1 \leq j \leq p_{q} .
$$

Since $\mu\left(K_{q}\right)=1$, from (17) we deduce $\mu_{1}\left(K_{q}\right)=\mu_{2}\left(K_{q}\right)=1$. Applying Lemma 9 we obtain

$$
\mu_{1}\left(f^{j}\left(\bar{I}_{q}\right)\right)=\mu_{2}\left(f^{j}\left(\bar{I}_{q}\right)\right)=\frac{1}{p_{q}} \quad \forall 1 \leq j \leq p_{q} .
$$

So, Lemma 4 implies $d\left(\mu_{1}, \mu\right)<\varepsilon$, and $d\left(\mu_{2}, \mu\right)<\varepsilon$. Since $\varepsilon>0$ is arbitrary, we conclude that $\mu=\mu_{1}=\mu_{2}$; hence $\mu$ is ergodic.

\section{All ergodic measures are pSEudo-Physical.}

Definition 19. Let $q \geq 1$ and $x_{0}$ be a periodic point with period $r \geq 1$. We call the (invariant) measure

$$
\nu=\frac{1}{r} \sum_{j=0}^{r-1} \delta_{f^{j}\left(x_{0}\right)} \in \operatorname{Per}_{f}
$$

a $q$-shrinked periodic measure, if there exists some periodic shrinking set $I$, with diameter smaller than $1 / q$, with period $p \geq 1$ such that $\nu$ is supported on $K:=\bigcup_{j=1}^{p} f(\bar{I})$. From the definition of periodic shrinking set, the period $p$ must divide $r$. We denote by $\operatorname{Shr}_{q} \mathrm{Per}_{f}$ the set of $q$-shrinked periodic measures.

We say that an invariant measure $\mu$, is $\varepsilon$-approached by q-shrinked periodic measures if there exists $\nu \in \operatorname{Shr}_{q} \operatorname{Per}_{f}$ such that $d(\mu, \nu)<\varepsilon$. We denote by $\mathrm{AShr}_{\varepsilon, q} \mathrm{Per}_{f}$ the set of measures that are $\varepsilon$-approached by $q$-shrinked periodic measures.

Theorem 20. For any map $f \in \mathcal{C}(M)$ (resp. $f \in \mathcal{H}(M)$ )

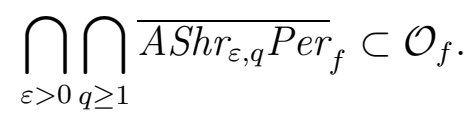

Proof. Fix $\varepsilon>0$, and choose $q \geq 1$ as in Lemma 4, such that $1 / q<\varepsilon$. For any $\mu_{q} \in \mathrm{AShr}_{1 / q, q} \mathrm{Per}_{f}$, denote by $\nu_{q}$ a measure in $\operatorname{Shr}_{q} \operatorname{Per}_{f}$ such that

$$
d\left(\mu_{q}, \nu_{q}\right)<1 / q<\varepsilon .
$$

Consider the periodic shrinking set $I=I\left(\nu_{q}\right)$ and the compact set $K$ for $\nu_{q}$ from Definition 19. From the definition of periodic shrinking set, any point $x \in I$ satisfies $f^{n}(x) \subset K=\bigcup_{j=0}^{p-1} f^{j}(\bar{I})$ for all $n \geq 0$. So, 
any measure $\mu_{x} \in p \omega(x)$ is supported on $K$. Also $\nu_{q}$ is supported on $K$. Thus, applying Lemma 9, we deduce that

$$
\mu_{x}\left(f^{j}(\bar{I})\right)=\nu_{q}\left(f^{j}(\bar{I})\right)=\frac{1}{p} \forall 1 \leq j \leq p ; \quad \operatorname{diam}\left(f^{j}(\bar{I})\right)<\frac{1}{q} .
$$

Now, from Lemma 4, we obtain $d\left(\nu_{q}, \mu_{x}\right)<\varepsilon$ for all $\mu_{x} \in p \omega(x)$, for all $x \in I$. Thus, for any $x \in I$

$$
d\left(p \omega(x), \nu_{q}\right)<\varepsilon \text {, hence } d\left(p \omega(x), \mu_{q}\right)<2 \varepsilon \text {. }
$$

Note that when we vary the value of $\varepsilon>0$, the value of $q$, and thus also the measures $\nu_{q}$ and $\mu_{q}$ and the set $I$, may change. So, from the above inequality we can not deduce that each $\mu_{q}$ is pseudo-physical. Nevertheless, we have proved that for any fixed value of $\varepsilon>0$ there exists $q \geq 1$ such that Inequality (18) holds for all $\mu_{q} \in \mathrm{AShr}_{1 / q, q} \mathrm{Per}_{f}$.

Now, consider any measure $\mu_{q}^{\prime} \in \overline{\mathrm{AShr}_{1 / q, q} \operatorname{Per}_{f}}$. Thus, there exists $\mu_{q} \in \mathrm{AShr}_{1 / q, q} \operatorname{Per}_{f}$ such that

$$
d\left(\mu_{q}^{\prime}, \mu_{q}\right)<\varepsilon
$$

Combining this with (18) we deduce that, for all $\varepsilon>0$ there exists $q \geq 1$ such that, for any measure $\mu_{q}^{\prime} \in \overline{\mathrm{AShr}_{1 / q, q} \operatorname{Per}_{f}}$ there exists an open set $I$ (the periodic shrinking set $I\left(\nu_{q}\right)$ for the measure $\nu_{q}$ associated to $\mu_{q}^{\prime}$ ) such that

$$
d\left(p \omega(x), \mu_{q}^{\prime}\right)<3 \varepsilon \quad \forall x \in I .
$$

So, if $\mu \in \bigcap_{\varepsilon>0} \bigcap_{q \geq 1} \overline{\operatorname{AShr}_{\varepsilon, q} \operatorname{Per}_{f}}$, then, for all $\varepsilon>0$ there exists an open set $I$ satisfying assertion (19). Thus $\operatorname{Leb}\left(A_{3 \varepsilon}(\mu)\right) \geq \operatorname{Leb}(I)>0$ for all $\varepsilon>0$; hence $\mu \in \mathcal{O}_{f}$, as wanted.

The last ingredient of the proof of Theorem 1 is the following theorem.

Theorem 21. For a typical map $f \in \mathcal{C}(M)$ (resp. $f \in \mathcal{H}(M)$ ),

$$
\operatorname{Per}_{f} \subset \bigcap_{q \geq 1} \overline{A S h r_{\varepsilon, q} \operatorname{Per}_{f}} \forall \varepsilon>0 .
$$

Before proving Theorem 21, let us introduce the following definition:

Definition 22. Fix $q, r \in \mathbb{N}^{+}$. A good $q, r$-covering $\mathcal{U}_{q, r}$ for $f \in \mathcal{C}(M)$ (resp. $\mathcal{H}(M)$ ), is a finite family of open simplexes (i.e., the interiors of simplexes) such that

(1) $\mathcal{U}_{q, r}$ covers the compact set $\operatorname{Per}(f, r):=\left\{x \in M: f^{r} x=x\right\}$.

(2) $\operatorname{diam}\left(U_{i}\right)<1 / q$ for any $U_{i} \in \mathcal{U}_{q, r}$.

(3) For any $U_{i} \in \mathcal{U}_{q, r}$, there exists a periodic shrinking set $I_{i}$, with period $p_{i} \leq r$, with $p_{i}$ that divides $r$, such that $\bar{I}_{i} \subset U_{i}$. 
We call a map $f \in \mathcal{C}(M)$ (resp. $\mathcal{H}(M)$ ) a good $q, r$-covered map, if there exists a good $q, r$-covering $\mathcal{U}_{q, r}$ for $f$. We denote by $\mathcal{P}_{q, r} \subset \mathcal{C}(M)$ (resp. $\mathcal{H}(M))$ the set of all good $q, r$-covered maps.

Proof of Theorem [21. We claim that, for fixed $q, r \geq 1$, the set $\mathcal{P}_{q, r}$ is open in $\mathcal{C}(M)$. Fix $f \in \mathcal{P}_{q, r}$, and denote its good $q, r$-covering by $\mathcal{U}_{q, r}=$ $\left\{U_{1}, U_{2}, \ldots, U_{h}\right\}$. The compact set $K=M \backslash \bigcup_{i=1}^{h} U_{i}$ does not intersect the compact set $\left\{f^{r}(x)=x\right\}$. Let us prove that for all $g \in \mathcal{C}(M)$ (resp. $\mathcal{H}(M)$ ) close enough to $f$, the same compact set $K$ (defined for the same covering $\left.\mathcal{U}_{q, r}\right)$ does not intersect $\left\{g^{r}(x)=x\right\}$. In fact, the real function $\phi_{f}(\cdot):=\operatorname{dist}\left(f^{r}(\cdot), \cdot\right)$ depends continuously on $f$. Since $\min _{x \in K} \phi_{f}(x)>0$, we deduce $\min _{x \in K} \phi_{g}(x)>0$ for all $g \in \mathcal{C}(M)$ (resp. $\mathcal{H}(M)$ ) close enough to $f$. In other words, $\mathcal{U}_{q, r}$ also covers the fixed points of $g^{r}$. Thus, the good $q, r$-covering of $f$, is also a covering satisfying conditions (1) and (2) of Definition 22, for any $g \in \mathcal{C}(M)$ (resp. $\mathcal{H}(M)$ ) close enough to $f$. Now, let us prove that Condition (3) for $g$ is satisfied by the same covering $\mathcal{U}_{q, r}$, provided that $g$ is close enough to $f$. Consider a $f$-shrinking periodic set $I_{i} \subset U_{i} \in \mathcal{U}_{q, r}$, of period $p_{i}$. Now $I_{i}$ is a periodic shrinking set with the same period $p_{i}$ for all $g$ sufficient close to $f$. Since the family $\left\{I_{i}\right\}_{1 \leq i \leq h}$ of shrinking periodic sets to be preserved is finite, we conclude that (3) is also satisfied for any $g$ sufficiently close to $f$ and thus $\mathcal{P}_{q, r}$ is open in $\mathcal{C}(M)$ (resp. $\mathcal{H}(M))$.

In Lemma 23, we will prove that $\mathcal{P}_{q, r}$ is dense in $\mathcal{C}(M)($ resp. $\mathcal{H}(M))$. Let us conclude the proof of Theorem 21 assuming that Lemma 23 is proven. Observe that, for fixed $q, r \geq 1$, any $f \in \mathcal{P}_{q, r}$ has the following property: any point $x_{0}$ fixed by $f^{r}$ (in particular any periodic point $x_{0}$ of period $r$ ) is $(1 / q)$-near all the points of a periodic shrinking set $I_{0}$ with diameter smaller than $1 / q$, and with period $p_{0} \leq r, p_{0}$ dividing $r$.

Besides, any periodic shrinked set of period $p_{0}$ has at least one periodic point $y_{0}$, fixed by $f^{p_{0}}$. We deduce that $I_{0}$, whose diameter is smaller than $1 / q$, contains a periodic point $y_{0}$. Using the definition of the set of measures $\mathrm{Shr}_{q} \mathrm{Per}_{f}$, we summerize this assertion as follows:

$$
\text { for all } x_{0} \text { with period } r \text {, there exists }
$$

$$
\nu_{0}:=\frac{1}{r} \sum_{j=0}^{r-1} \delta_{f^{j}\left(y_{0}\right)} \in \operatorname{Shr}_{q} \operatorname{Per}_{f} \text {, with } \operatorname{dist}\left(y_{0}, x_{0}\right)<1 / q
$$

For $r \geq 1$ fixed, consider $f \in \bigcap_{q \geq 1} \mathcal{P}_{q, r}$. Let $\mu_{0}:=\frac{1}{r} \sum_{j=0}^{r-1} \delta_{f^{j}\left(x_{0}\right)}$. Fix $\varepsilon>0$ and choose $q^{\prime} \geq 1$ as in Lemma 4. Since $f^{j}$ is continuous for each $j$, we can find a $q>q^{\prime}$ so that if $\operatorname{dist}(x, y)<1 / q$, then 
$\operatorname{dist}\left(\delta_{f^{j} x}, \delta_{f^{j} y}\right)<1 / q^{\prime}$ for all $j \in\{0,1, \ldots, r\}$. Then Lemma 4 implies that $d\left(\mu_{0}, \nu_{0}\right)<\varepsilon$.

We have shown that for any given periodic orbit $\left\{f^{j}\left(x_{0}\right)\right\}_{0 \leq j \leq r-1}$ of period $r$, the distance between the periodic measure supported on it, and some measure $\nu_{q} \in \operatorname{Shr}_{q} \operatorname{Per}_{f}$, for all $q$ large enough, is smaller than $\varepsilon$. In other words, any periodic measure supported on a periodic orbit of period $r$, belongs to $\bigcap_{q>1} \overline{\mathrm{AShr}_{\varepsilon, q} \mathrm{Per}_{f}}$ for all $\varepsilon>0$.

Finally, if $f \in \mathcal{P}:=\bigcap_{r \geq 1} \bigcap_{q>1} \mathcal{P}_{q, r}$, then all its periodic measures (supported on periodic orbits of any period $r$ ) will belong to $\overline{\operatorname{AShr}_{\varepsilon, q} \operatorname{Per}_{f}}$ for all $q \geq 1$ and for all $\varepsilon>0$. In brief, if $f \in \mathcal{P}$, then

$$
\bigcap_{q \geq 1} \operatorname{Per}_{f} \subset \overline{\operatorname{AShr}_{\varepsilon, q} \operatorname{Per}_{f}} \quad \forall \varepsilon>0 .
$$

As $\mathcal{P}_{q, r}$ is open and dense in $\mathcal{C}(M)$ (resp. $\mathcal{H}(M)$ ), the maps $f \in \mathcal{P}$ are typical. This ends the proof of Theorem 21, provided that Lemma 23 is proven.

Lemma 23. For each $q, r \geq 1$, the set $\mathcal{P}_{q, r}$ is dense in $\mathcal{C}(M)$ (resp. $\mathcal{H}(M))$.

Proof. We will use the notation that we defined in Subsection 2.2. Fix $f \in \mathcal{C}(M)(\operatorname{resp} . \mathcal{H}(M))$ and $\varepsilon>0$. Consider $0<\delta:=\frac{1}{q^{\prime}}<\min \left\{\frac{1}{q}, \frac{\varepsilon}{2}\right\}$ such that $\operatorname{dist}(x, y)<\delta$ implies $\operatorname{dist}(f(x), f(y))<\varepsilon$. We wish to $\varepsilon$ perturb $f$ into new map $g \in \mathcal{P}_{q, r}$. Consider a triangulation $\mathcal{T}:=$ $\left\{\bar{T}_{1}, \ldots, \bar{T}_{h}\right\}$ of $M$ such that the diameters of all the simplexes $\bar{T}_{i}$ are at most $\delta / 3$. By modifying $\mathcal{T}$ we can suppose that if $\bar{T}_{i} \cap \operatorname{Per}(f, r) \neq \emptyset$ then $T_{i} \cap \operatorname{Per}(f, r) \neq \emptyset$, where $T_{i}=\operatorname{int}\left(\bar{T}_{i}\right)$; and furthermore, for some $x_{i} \in T_{i} \cap \operatorname{Per}(f, r)$ its orbit $\left\{x_{i}, f\left(x_{i}\right), \ldots, f^{r-1}\left(x_{i}\right)\right\} \cap \partial \mathcal{T}=\emptyset$. Indeed, suppose some $\bar{T}_{i}$ does not satisfy these conditions. If no point of $\left\{x: f^{r} x=x\right\}$ is in the interior of $\bar{T}_{i}$ but some is in $\partial T_{i}$, then we can modify the triangulation by first slightly moving one corner of $\bar{T}_{i}$ so that in the resulting triangulation some point $x_{i} \in \operatorname{Per}(f, r)$ belongs to the interior $T_{i}$, and all the other simplexes of $\mathcal{T}$ which satisfied this condition before, still satisfy it.

If the orbit of $x_{i}$ does not lay in the interior of the simplexes, let $1 \leq j \leq r-1$ be the first time that $f^{j}\left(x_{i}\right) \in \partial \mathcal{T}$. Again we modify the triangulation by slightly moving one corner of the triangulation so that in the modified triangulation $f^{j}\left(x_{i}\right) \notin \partial \mathcal{T}$, and all the other simplexes of $\mathcal{T}$ which satisfied this condition before, still satisfy it. Repeat this procedure a finite number of times to produce the desired triangulation. We can assume that the perturbations are so small that in the resulting triangulation the diameters of all the simplexes are at most $\delta / 2$. 
Let $\mathcal{T}^{(1)}:=\left\{\bar{T}_{1}^{(1)}, \ldots, \bar{T}_{l}^{(1)}\right\}:=\left\{\bar{T}_{i} \in \mathcal{T}: T_{i} \cap \operatorname{Per}(f, r) \neq \emptyset\right\}$ and for each $i$ let $x_{i}$ be a point in $T_{i}^{(1)} \cap \operatorname{Per}(f, r)$. Using $x_{i}$ as a centroid, consider a real number $\lambda_{\bar{T}_{i}^{(1)}}>1$ near enough 1 , such that $\lambda_{\bar{T}_{i}^{(1)}} \bar{T}_{i}^{(1)}$ is well defined. Let $\lambda_{\mathcal{T}}:=\min \left\{\lambda_{T_{i}^{(1)}}: 1 \leq i \leq l\right\}$. For $\lambda \in\left(1, \lambda_{\mathcal{T}}\right)$ we have $x_{i} \in T_{i} \subset \bar{T}_{i} \subset \lambda T_{i}$, and $\left\{\lambda T_{1}, \ldots, \lambda T_{l}\right\}$ is an open cover of $\operatorname{Per}(f, r)$. We choose such a value of $\lambda_{\max } \in\left(1, \lambda_{\mathcal{T}}\right)$, so close to 1 such that besides, each of the points $x_{i}$ does not lie inside $\lambda \bar{T}_{j}$ for any $j \neq i$, and furthermore such that the diameters of the $\lambda T_{i}$ are at most $\delta$. For the rest of the proof we fix such a value of $\lambda \in\left(1, \lambda_{\mathcal{T}}\right)$.

Denote

$$
F:=\left\{x_{i}: 1 \leq i \leq l\right\}, \quad F^{r}:=\bigcup_{j=0}^{r-1} f^{j}(F) .
$$

Hence $F$ is consisits of exactly $l$ different points that are fixed by $f^{r}$, and $F^{r}$ consists of at most $r l$ different points, also fixed by $f^{r}$.

We will define an homeomorphism $h: M \mapsto M$. Consider a chart $\left(U_{\alpha}, \phi_{\alpha}\right)$ such that $\lambda \bar{T}_{i} \subset U_{\alpha}$. Choose $\eta>0$ so small such that for each $x \in F^{r}$ the solid ball $B\left(\phi_{\alpha}(x), \eta\right) \subset \mathbb{R}^{m}$ does not intersect $\phi_{\alpha}\left(U_{\alpha} \cap\right.$ $\partial(\lambda \mathcal{T})$ ), such that the diameter of $\mathbf{B}(x, \eta):=\phi^{-1}(B(x, \eta))$ is at most $\delta$, and such that the finite family $\left\{\mathbf{B}(x, \eta): x \in F^{r}\right\}$ is composed of pairwise disjoint sets. We define $h(y)=y$ if $\left.y \notin \bigcup_{x \in F^{r}} \mathbf{B}(x, \eta)\right)$. On the complement we define $h$ as follows. Fix $x \in F^{r}$, and consider polar/spherical coordinates $(s, \theta): s \in[0,1], \theta \in \mathbb{S}^{m}$ to describe the ball $B(x, \eta)$. Finally use $\phi_{\alpha}^{-1}$ to pull back these coordinates to $\mathbf{B}(x, \eta)$.

Fix a certain $k \geq 1$ sufficiently large (that will be chosen later), and define $h: \mathbf{B}(x, \eta) \mapsto \mathbf{B}(x, \eta)$ by setting $h(s, \theta):=\left(s^{k}, \theta\right)$. This construction for each point $x \in F^{r}$ completes the definition of the homeomorphism $h$. Thus if $f$ is continuous then so is

$$
g: f \circ h
$$

and if $f$ is an homeomorphism, then $g$ is also an homeomorphism.

Note that for any $y \in M$ the map $h$ satisfies

$$
\operatorname{dist}(y, h(y))<\delta \quad \text { and } \quad \operatorname{dist}\left(y, h^{-1}(y)\right)<\delta .
$$

Since $g=f \circ h$ and $\operatorname{dist}(h(y), y)<\delta$ we have

$$
\rho(f, g)<\varepsilon .
$$

If besides $f$ is an homeomorphism, then $g^{-1}=h^{-1} \circ f^{-1}$ and

$$
\operatorname{dist}\left(g^{-1}(y), f^{-1}(y)\right)=\operatorname{dist}\left(h^{-1}(z), z\right)<\delta<\varepsilon .
$$

Thus

$$
\rho\left(g^{-1}, f^{-1}\right)<\varepsilon
$$


We have proved that $g$ is an $\varepsilon$-perturbation of $f$ in $\mathcal{C}(M)$ (resp. $\mathcal{H}(M)$ ). Now, to end the proof of the lemma, it is enough to choose $k$ such that the set $\mathbf{B}(x, \eta)$ contains a periodic shrinking set of period that divides $r$ for each point $x$ of the finite set $F^{r}$.

From the above construction, we have $h(0, \theta)=0$. Therefore, $g(x)=$ $g(0, \theta)=f(0, \theta)=f(x)$ for all $x \in F^{r}$; thus each point $x$ of $F^{r}$ will be fixed by the map $g^{r}$.

We claim that if we choose $k$ large enough, then $g$ has a shrinking set around the point $x \in T_{i} \cap F^{r}$ contained in $\mathbf{B}(x, \eta)$. We consider the $(s, \theta)$ coordinates in the set $\mathbf{B}(x, \eta)$. Fix $\eta_{1} \in(0, \eta)$. For each $j=0,1, \ldots, r-1$ choose a simplex $\bar{I}_{j}$ containing $f^{j}(x)$ such that $\bar{I}_{j} \subset \mathbf{B}\left(f^{j}(x), \eta_{1}\right)$. Next choose $\eta_{2} \in\left(0, \eta_{1}\right)$ small enough such that $f\left(\mathbf{B}\left(f^{j} x, \eta_{2}\right)\right) \subset I_{j+1}$ for $j=0,1, \ldots, r-1$. In these coordinates $\mathbf{B}\left(x, \eta_{i}\right)$ is given by $\left\{(s, \theta): s \in\left[0, \eta_{i}\right]\right\}$. Choose $k>1$ so that $\eta_{1}^{k}<\eta_{2}$. For each $j=1, \ldots, r$ we have

$$
g\left(\bar{I}_{j}\right) \subset g\left(\mathbf{B}\left(f^{j} x, \eta_{1}\right)\right)=f \circ h\left(\mathbf{B}\left(f^{j} x, \eta_{1}\right)\right) \subset f\left(B^{j} x, \eta_{2}\right) \subset I_{j+1} .
$$

Thus the simplexes $\bar{I}_{j}$ are the desired shrinking sets. This finishes the proof of Lemma 23

\subsection{End of the proof of Theorem 1.}

Proof. In Corollary 18 we have proved that $\mathcal{O}_{f} \subset \overline{\mathcal{E}_{f}}=\overline{\operatorname{Per}}_{f}$. Combining Theorems 20 and 21, we deduce that

$$
\operatorname{Per}_{f} \subset \bigcap_{\varepsilon>0} \bigcap_{q \geq 1} \overline{\operatorname{AShr}_{\varepsilon, q} \operatorname{Per}_{f}} \subset \mathcal{O}_{f}
$$

Since $\mathcal{O}_{f}$ is closed, we conclude that $\mathcal{O}_{f}=\overline{\mathcal{E}_{f}}=\overline{\mathrm{Per}}_{f}$, as wanted.

\section{REFERENCES}

[AA] F. Abdenur and M. Andersson, Ergodic theory of generic continuous maps, Comm. Math. Phys. 318 (2013), no. 3, 831-855.

[AHK] E. Akin, M. Hurley, and J.A. Kennedy. Dynamics of topologically generic homeomorphisms, Mem. Amer. Math. Soc., 164(783): viii+130, 2003.

[AP] V.S. Alpern, and S. Prasad, Typical dynamics of volume preserving homeomorphisms, Volume 139 of Cambridge Tracts in Mathematics. Cambridge: Cambridge University Press, 2000

[AH] N. Aoki, and K. Hiraide, Topological Theory of Dynamical Systems: Recent Advances, North Holland 1994.

[AM] A. Arbieto and C.A. Morales, Expansivity of ergodic measures with positive entropy, ArXiv: 1110.5598 [math.DS], 2011

[CE1] E. Catsigeras and H. Enrich, SRB-like measures for $C^{0}$ dynamics, Bull. Pol. Acad. Sci. Math. 59 (2011), no. 2, 151-164.

[CE2] E. Catsigeras and H. Enrich, Equilibrium states and SRB-like measures of $C^{1}$-expanding maps of the circle, Port. Math. 69 (2012), no. 3, 193-212. 
[CCE1] E. Catsigeras, M. Cerminara, and H. Enrich, The Pesin entropy formula for $C^{1}$ diffeomorphisms with dominated splitting, Ergodic Theory Dynam. Systems 35 (2015) 737-761.

[CCE2] E. Catsigeras, M. Cerminara, and H. Enrich, Weak Pseudo-Physical Measures and Pesin's Entropy Formula for Anosov $C^{1}$ diffeomorphisms, Contemporary Mathematics 698 (2017) 69-89.

[CT] E. Catsigeras, and S. Troubetzkoy, Pseudo-physical measures for typical continuous maps of the interval, arXiv:1705.10133v1

[CS] Y. Coudene and B. Schapira, Generic measures for hyperbolic flows on noncompact spaces, Israel Journ. Math. 179 (2010), 157-172.

[H1] M. Hurley, On proofs of the $C^{0}$ general density theorem, Proc. Amer. Math. Soc., 124 (1996) 1305-1309.

[H2] M. Hurley, Properties of attractors of generic homeomorphisms, Ergodic Theory Dynam. Systems, 16 (1996) 1297-1310.

[GK] K. Gelfert and D. Kwietniak, On density of ergodic measures and generic points, Erg. Th. \& Dyn. Sys. 38 (2018) 1745-1767.

[KMOP] P. Koscielniak, M. Mazur, P. Oprocha and P. Pilarczyk, Shadowing is generic-a continuous map case, Discr. Contin. Dyn. Syst. 34 (2014) 3591-3609.

[L] J.M. Lee, Introduction to smooth manifolds Springer Verlag, 2003.

[M] J.Munkres, Obstructions to the smoothing of piecewise-differentiable homeomorphisms Annals Math. 72 (1960) 521-554.

[O] K. Odani, Generic homeomorphisms have the pseudo-orbit tracing property, Proc. Amer. Math. Soc. 110 (1990) 281-284.

[OU] J.C. Oxtoby, and S.M. Ulam, Measure-preserving homeomorphisms and metrical transitivity, Ann. of Math. (2) 42 (1941) 874-920.

[PP] S.Yu. Pilyugin, and O.B. Plamenevskaya, Shadowing is generic, Topology and its Applications 97 (1999) 253-266.

[W] J.H.C. Whitehead, On $C^{1}$-complexes, Annals Math. 41 (1940) 809-824.

[Y] K. Yano, Generic homeomorphisms of $S^{1}$ have the pseudo-orbit tracing property, J. Fac. Sci. Univ. Tokyo, Sect. IA Math. 34 (1987) 51-55.

Instituto de Matemática y Estadística "Prof. Ing. Rafael Laguardia" (IMERL), Universidad de la República, Av. Julio Herrera y Reissig 565, C.P. 11300, Montevideo, Uruguay

E-mail address: eleonora@fing.edu.uy

$U R L$ : http:/fing.edu.uy/ eleonora

Aix Marseille Univ, CNRS, Centrale Marseille, i2M, Marseille, FRANCE

postal address: I2M, Luminy, Case 907, F-13288 Marseille Cedex 9, FRANCE

E-mail address: serge.troubetzkoy@univ-amu.fr

URL: http://www.i2m.univ-amu.fr/perso/serge.troubetzkoy/ 